\title{
Valores y consumo de alcohol y tabaco en jóvenes universitarios
}

\author{
Rodríguez Aguilar Lucio ${ }^{1}$, De La Garza Guerrero Lidice Sarai ${ }^{2}$, Gricelda Yahibe Rodríguez Davalos ${ }^{3}$, \\ Alonso Castillo María Magdalena ${ }^{4}$, Guzmán Facundo Francisco Rafael ${ }^{5}$.
}

\section{RESUMEN}

Objetivos: conocer la relación que existe del perfil de valores y el consumo de alcohol y tabaco en 268 estudiantes del área de salud y de ingeniería. Material y métodos: el diseño del estudio fue de corte transversal y correlacional. Se utilizó el Cuestionario de Valores de Schwartz (SVS) y el Cuestionario de Identificación de los Trastornos por Uso del Alcohol (AUDIT). Resultados: se encontró que un 77,2\% ha consumido alcohol en el último año. En relación con el tabaco $30,6 \%$ ha consumido tabaco en el último año. Se mostró diferencia significativa del índice general de valores $(U=7571, p=.030)$ entre las carreras de enfermería e ingeniería, siendo mayor las medias en los universitarios de la carrera de enfermería. Así mismo las mujeres mostraron mayor puntuación en los valores que en los hombres $(U=7048, p=0,031)$, exceptuando el domino de valor poder. Se identificó que el valor de Hedonismo presenta efecto en el consumo de alcohol y tabaco. El valor Seguridad mostró efecto en el no consumo de alcohol y el valor de Conformidad mostró efecto en el no consumo de tabaco. Conclusiones: estos resultados permiten reflexionar sobre la integración de valores al desarrollar intervenciones de enfermería para la prevención del consumo de alcohol y tabaco en jóvenes universitarios.

PALABRAS CLAVES: valores, alcohol, tabaco, jóvenes. México.

\section{Values and alcohol and tobacco consumption in college students}

\section{SUMMARY}

Objectives: It was known the relationship Profile of values and consumption of alcohol and snuff 268 students in the area of health and engineering. Material and Methods: The study design was cross-sectional and correlational. Values Questionnaire Schwartz (SVS) and the Questionnaire of identification of the Alcohol Use Disorders (AUDIT) was used. Results: We found that $77.2 \%$ had consumed alcohol in the past year. Regarding the snuff snuff consumed $30.6 \%$ in the last year. significant difference in the overall index value $(U=7571, p=.030)$ were demonstrated between nursing and engineering careers, the average was higher in university nursing career. Likewise, women showed higher scores in values than males $(U=7048, p=0.031)$, except the power domain value. It was identified that the value of Hedonism has effect on the consumption of alcohol and snuff. The Security value showed no effect on alcohol consumption and the value of Conformity showed no effect on the consumption of snuff. Conclusions: these results allow considering integrating values in developing nursing interventions for prevention of alcohol and snuff among university students.

KEYWORDS: values, alcohol, snuff, youth. Mexico.

Maestro en Salud Pública, Universidad Autónoma de Nuevo León, Facultad de Enfermería. Monterrey, NL. México.

Maestra en Ciencias de Enfermería. Universidad Autónoma de Nuevo León, Facultad de Enfermería. Monterrey, NL. México.

Maestra en Ciencias. Universidad Autónoma de Nuevo León, Facultad de Enfermería. Monterrey, NL. México

Profesor de tiempo completo, Subdirectora de Postgrado e Investigación.

Doctor en Enfermería. Universidad Autónoma de Nuevo León, Facultad de Enfermería. Monterrey, NL. México. 


\section{INTRODUCCIÓN}

En la actualidad se está en un periodo de pesimismo y deshumanización donde los valores en los jóvenes se han ido perdiendo, la utilización de su tiempo disponible, además de tener algunos problemas en la ingesta abusiva de alcohol y otras drogas, están en un tipo de ausencia social, en el que los problemas de su entorno no parecen preocuparles y esto es evidencia de la falta de expresión de valores relacionados al no consumo de sustancias (1). El consumo de tabaco y alcohol como hábito social ha estado presente a lo largo de la historia de todas las sociedades. Las tendencias demográficas con respecto al consumo, indican que la edad de inicio del consumo de alcohol y tabaco es cada vez más temprana y su prevalencia se ha incrementado en los últimos años $(2,3)$.

El consumo de estas sustancias continúan siendo un grave problema de salud pública, especialmente entre los adolescentes, adultos jóvenes y las mujeres con una tendencia al incremento en la frecuencia de consumo (4). En este sentido el abuso de alcohol, por sí solo representa el 9\% del peso total de la enfermedad en México y el consumo de tabaco se mantienen como la primera causa de muerte prevenible a nivel mundial (5).

Se ha reportado la influencia de valores en el consumo de alcohol en los jóvenes universitarios, tales como el aumento del sentido de poder, el incremento de la sexualidad, el desenvolvimiento verbal y el buscar generar otros cambios fisiológicos agradables $(6,7)$. Los valores es un aspecto ineludible en la comprensión del comportamiento de la persona en el caso de las conductas de riesgo como es el consumo de alcohol y tabaco; al respecto, se refiere que la conducta para el consumo de alcohol y tabaco, no puede ser analizada sin considerar el sistema de valores humanos (8). En la literatura revisada, poco se conoce sobre el perfil de valores que tienen los jóvenes universitarios así como su relación con el consumo de alcohol y tabaco. Por tal motivo el propósito del presente estudio es conocer la relación que existe del perfil de valores y el consumo de alcohol y tabaco en estudiantes universitarios del área de salud y de ingeniería con la finalidad de profundizar el conocimiento y en un futuro, generar áreas de oportunidad, para la aplicación de intervenciones preventivas de enfermería.

\section{Valores}

Para fines de este estudio se tomaron en cuenta las posiciones teóricas de Schwartz (9), en la versión más reciente de su teoría transcultural de valores, articula los valores en torno a su objetivo instrumental y terminal, que expresan intereses (individuales, colectivos ó ambos) pertenecientes a un dominio motivacional y es evaluado en un rango de importancia como principio rector de vida (10).

Schwartz (10) reveló la existencia de diez tipos motivacionales de los valores, los cuales son hedonismo (valores cuya meta es la búsqueda de placer), autorrealización (éxito personal alcanzado mediante el esfuerzo y la capacidad), poder social (búsqueda de prestigio y control social sobre las personas y los recursos), autodeterminación (valores cuya meta es la independencia de pensamiento), conformidad (control de los impulsos y comportamientos de acuerdo a las normas sociales), benevolencia (preocupación e interés por el bienestar de las personas más cercanas afectivamente), universalismo (valores tendientes a preocuparse por el bien común), tradición (respeto y aceptación de las costumbres de la sociedad), seguridad (resguardar y preservar la integridad personal y de la sociedad) y estimulación (búsqueda de excitación y cambio para mantener un buen nivel de funcionamiento).

\section{MATERIAL Y MÉTODOS}

El diseño de estudio fue tipo descriptivo de correlación (11). La población de estudio fueron jóvenes universitarios, de ambos sexos,inscritos en dos facultades de una universidad pública del área metropolitana del estado de Nuevo León, 1,818 universitarios del área de salud y 2,054 universitarios del área de ingeniería. El muestreo fue probabilístico estratificado. El cálculo de la muestra se obtuvo a través del paquete estadístico n'QueryAdvisor Versión 4,0. La muestra calculada fue de 126 jóvenes participantes de la carrera enfermería y 142 de la carrera de ingeniería.

Se utilizó una Cédula de Datos Personales (CDP) y dos instrumentos para la medición de las variables de estudio: el Cuestionario de Valores de Schwartz (SVS) y el Cuestionario de Identificación de los Trastornos debidos al Consumo de Alcohol (AUDIT).

El SVS permite calcular la importancia dada por las personas a los valores, y describe las posibles conductas que poseen. El cuestionario consta de 21 (de un total de 57) valores y su formulación como principios que guían la vida del sujeto. Se trata de una escala de tipo Likert en la cual la numeración va desde 1 que corresponde a no importante hasta el 7 que corresponde a muy importante, es decir cuanto mayor es el número, más importante es el 
valor como principio guía fundamental en la vida.

En relación a la evaluación, se cuenta con 10 dimensiones, en donde al dominio de Conformidad le corresponden las preguntas 11, 20, 40 y 47, al dominio de Tradición le corresponden las preguntas $18,32,36,44$ y 51 , al dominio de Benevolencia le corresponden las preguntas 33, 45, 49, 52 y 54 , al dominio de Universalidad le corresponden las preguntas $1,17,24,26,29,30,35$ y 38 , al dominio de Individualismo le corresponden las preguntas 5, 16, 31, 41 y 53, al dominio de Estimulación le corresponden las preguntas 9, 25 y 37, al dominio de Hedonismo le corresponden las preguntas 4, 50 y 57, al dominio de Logro le corresponden las preguntas $34,39,43$ y 55 , al dominio de Poder le corresponden las preguntas 3, 12, 27 y 46 y por último al dominio de Seguridad le corresponden las preguntas $8,13,15,22$ y 56 .Así mismo, los reactivos del 1 al 30 corresponden a los valores terminales y de los ítems del 31 al 57 corresponden a los valores instrumentales. Para fines de este estudio cada dimensión se convirtió en índice de tal forma que se obtuvieron valores de 0 a 100 , lo que indica que a mayor puntuación mayor son los valores. Así mismo se obtuvo una confiabilidad de $\alpha=0,93$.

Para medir la variable de Consumo de Alcohol se utilizó el AUDIT $(12,13)$, cuestionario autoadministrado, útil y con validez transcultural diseñada para detectar problemas leves y moderados relacionados con el alcohol. Consta de 10 reactivos que examinan el consumo de alcohol durante los últimos doce meses y sus consecuencias. Contiene tres dominios, los reactivos del 1 al 3 determinan la cantidad y frecuencia del consumo; los reactivos del 4 al 6 , exploran la posibilidad de que exista dependencia al alcohol y los reactivos del 7 al 10 exploran el consumo dañino de alcohol. Tiene un valor mínimo de 0 y un máximo de 40 puntos, donde a mayor puntaje existe mayor consumo de alcohol. Si se obtiene un valor de 1 a 3 se considera un consumo sin riesgo (consumo sensato), si se obtiene una puntuación por encima de 8 puntos se considera un consumo de riesgo elevado o consumo dañino. Para esta muestra obtuvo una confiabilidad de $\alpha=.82$.

El estudio fue revisado y autorizado por las comisiones de ética y de investigación de la Facultad de Enfermería de la UANL, así mismo se solicitó la autorización de los directivos de las facultades donde fue realizado el estudio. Los estudiantes fueron seleccionados aleatoriamente, posteriormente, los jóvenes que aceptaron participar, se les proporcionó el consentimiento informado. El estudio se orientó a lo estipulado en el Reglamento de la
Ley General de Salud en Materia de Investigación para la Salud (14). Los datos fueron procesados en el programa estadístico StatisticalPackageforthe Social Sciences (SPSS) versión 17 para Windows.

\section{RESULTADOS}

Los datos sociodemográficos de los participantes del presente estudio, en cuanto a sexo predominó el sexo masculino $(63,1 \%)$, el $31,3 \%$ de la población total cuentan con pareja y respecto a la ocupación un 19,8\% trabaja y estudia, el resto solo estudia.

En la tabla 1, se puede observar que los participantes tenían en promedio 19,68 años de edad. El índice de Valores en su escala total presentó un promedio de 75,83( $D E$ =9.65), para el índice de valores terminales e instrumentales se observa un promedio de 71,83 y 79,01 ¿Cuál es la medida? ( $D E=10,12 ; D E=10,24)$ respectivamente. Cabe destacar que los dominios de valores con mayor puntuación fueron conformidad, benevolencia, logro, universalidad e individualismo.

Respecto al Cuestionario de Identificación de Trastornos por Uso del Alcohol (AUDIT), el promedio de la sumatoria fue de 5.33. Los resultados de la prueba de Kolmogorov-Smirnov indican que la mayoría de las variables continuas no presentan distribución normal $(p<0,05)$, por lo que se decidió utilizar pruebas no paramétricas para dar cumplimiento al objetivo del estudio.

\section{Perfil de valores en universitarios}

La tabla 2, muestra diferencia significativa del índice general de valores $(U=7571, p=.030)$ entre las carreras de enfermería e ingeniería, siendo mayor las medias en los universitarios de la carrera de enfermería $(=76,64$, $M d n=77,19)$ que en la carrera de ingeniería $(=73,93$, $M d n=74,70)$; así también se muestran los dominios que mostraron diferencias significativas, los resultados muestran medias y medianas mayores en los estudiantes de enfermería.

La tabla 3, muestra las diferencias de los valores por sexo; en su índice general de valores, esta diferencia fue significativa $(U=7048, p=0,031)$, siendo mayor en el sexo femenino $(=76,79, M d n=77,19)$. Así mismo se muestran los dominios que mostraron diferencias significativas, cabe destacar que la mayoría de los dominios fueron mayor en la mujeres que en los hombres, sin embargo en el dominio de valor poder fue más alto en los hombres ( 
Tabla 1. Descripción de perfil de valores, consumo de alcohol (AUDIT) y Prueba de Kolmogorov-Smirnovcon corrección de Lilliefors.

\begin{tabular}{|c|c|c|c|c|c|c|c|}
\hline Variable & $n$ & & $D E$ & $\begin{array}{l}\text { Val } \\
\text { Min }\end{array}$ & $\begin{array}{l}\text { Val } \\
\operatorname{Max}\end{array}$ & $D^{a}$ & $p$ \\
\hline Edad & $268^{a}$ & 19,68 & 1,53 & 18,0 & 27,0 & 3,55 & ,001 \\
\hline Índice de valores & $268^{\mathrm{a}}$ & 75,83 & 9,65 & 47,95 & 95,91 & 0,56 & ,905 \\
\hline $\begin{array}{l}\text { Índices de dominios } \\
\text { Individualismo }\end{array}$ & $268^{\mathrm{a}}$ & 76,30 & 11,28 & 36,11 & 100,00 & 1,54 &, 017 \\
\hline Estimulación & $268^{a}$ & 41,21 & 13,26 & 5,56 & 100,00 & 1,77 & ,004 \\
\hline Hedonismo & $268^{\mathrm{a}}$ & 75,91 & 17,04 & 22,22 & 100,00 & 1,93 & ,001 \\
\hline Logro & $268^{\mathrm{a}}$ & 80,91 & 12,31 & 44,44 & 100,00 & 1,63 &, 010 \\
\hline Poder & $268^{a}$ & 52,28 & 18,97 & 0,00 & 100,00 & 1,32 &, 060 \\
\hline Seguridad & $268^{\mathrm{a}}$ & 71,89 & 11,81 & 40,48 & 100,00 & 0,99 & ,271 \\
\hline Conformidad & $268^{\mathrm{a}}$ & 88,75 & 11,97 & 50,00 & 100,00 & 2,97 & ,001 \\
\hline Tradición & $268^{\mathrm{a}}$ & 71,34 & 15,62 & 30,00 & 100,00 & 1,56 &, 015 \\
\hline Benevolencia & $268^{\mathrm{a}}$ & 82,17 & 12,35 & 46,30 & 100,00 & 1,95 &, 001 \\
\hline Universalidad & $268^{\mathrm{a}}$ & 77,46 & 13,15 & 29,63 & 100,00 & 1,53 & ,018 \\
\hline Sumatoria de AUDIT & $207^{b}$ & 5,33 & 4,97 & 0,16 & 0,18 & 2,63 &, 001 \\
\hline
\end{tabular}

Nota: ${ }^{\mathrm{a}} n=$ muestra total, ${ }^{\mathrm{b}} n=$ consumidores de alcohol en el último año, $=$ media, $D E=$ Desviación Estándar, $D^{a}=$ prueba de normalidad, $p=$ significancia.

Tabla 2. Prueba de U de Mann-Whitney para las variables de valores por carrera.

\begin{tabular}{lrrrrr}
\hline & & $M d n$ & $D E$ & $U$ & $p$ \\
\hline Índice de Valores & & & & & \\
$\quad$ Enfermería & 76,64 & 77,19 & 9,80 & 7571,0 &, 030 \\
$\quad$ Ingeniería & 73,99 & 74,70 & 9,38 & & \\
Índice de Valores Instrumentales & & & & & \\
$\quad$ Enfermería & 80,62 & 82,09 & 10,20 & 7397,0 &, 014 \\
$\quad$ Ingeniería & 77,59 & 78,39 & 10,09 & & \\
Índice del Dominio de Individualidad & & & & & \\
$\quad$ Enfermería & 77,84 & 80,55 & 10,76 & 7706,5 &, 050 \\
$\quad$ Ingeniería & 74,94 & 76,38 & 1,58 & & \\
Índice del Dominio de Conformidad & & & & & \\
$\quad$ Enfermería & 90,77 & 95,83 & 11,76 & 6894,0 &, 001 \\
$\quad$ Ingeniería & 86,97 & 87,50 & 11,91 & & \\
Índice del Dominio de Tradición & & & & & \\
$\quad$ Enfermería & 75,58 & 76,66 & 15,53 & 6262,5 &, 001 \\
$\quad$ Ingeniería & 67,58 & 66,66 & 14,76 & & \\
Índice del Dominio de Benevolencia & & & & & \\
$\quad$ Enfermería & 84,31 & 87,03 & 11,66 & 7272,5 & \multirow{2}{*}{008} \\
Ingeniería & 80,28 & 83,33 & 12,68 & & \\
Índice del Dominio de Universalidad & & & & & \\
$\quad$ Enfermería & 79,74 & 83,33 & 13,33 & 7145,5 & \multirow{2}{*}{004} \\
Ingeniería & 75,44 & 76,85 & 12,70 & & \\
\hline
\end{tabular}

Nota: $=$ media, $M d n=$ mediana, $U=\mathrm{U}$ de Mann-Whitney, $p=$ significancia. 
Tabla 3. Prueba de U de Mann-Whitney para la variable de valores por género

\begin{tabular}{|c|c|c|c|c|c|}
\hline & & $M d n$ & $D E$ & $U$ & $p$ \\
\hline \multicolumn{6}{|c|}{ Índice total de Valores } \\
\hline Masculino & 74,32 & 74,85 & 9,41 & 7048,0 & ,031 \\
\hline Femenino & 76,79 & 77,19 & 9,91 & & \\
\hline \multicolumn{6}{|c|}{ Índice de Valores Terminales } \\
\hline Masculino & 70,89 & 71,11 & 9,89 & 7076,0 & ,035 \\
\hline Femenino & 73,45 & 73,33 & 10,35 & & \\
\hline \multicolumn{6}{|c|}{ Índice de Valores Instrumentales } \\
\hline Masculino & 78,15 & 78,39 & 10,05 & 7085,0 & ,036 \\
\hline Femenino & 80,50 & 82,09 & 10,44 & & \\
\hline \multicolumn{6}{|c|}{ Índice del Dominio de Poder } \\
\hline Masculino & 54,89 & 53,33 & 18,06 & 6619,5 & ,004 \\
\hline Femenino & 47,84 & 46,66 & 19,75 & & \\
\hline \multicolumn{6}{|c|}{$\begin{array}{l}\text { Índice del Dominio de } \\
\text { Conformidad }\end{array}$} \\
\hline Masculino & 86,88 & 87,50 & 12,55 & 6254,5 &, 001 \\
\hline Femenino & 91,96 & 95,83 & 10,21 & & \\
\hline \multicolumn{6}{|c|}{ Índice del Dominio de Tradición } \\
\hline Masculino & 68,59 & 70,00 & 15,75 & 6075,0 & ,001 \\
\hline Femenino & 76,02 & 76,66 & 14,29 & & \\
\hline \multicolumn{6}{|c|}{$\begin{array}{l}\text { Índice del Dominio de } \\
\text { Benevolencia }\end{array}$} \\
\hline Masculino & 80,25 & 83,33 & 12,40 & 6250,5 & ,001 \\
\hline Femenino & 85,46 & 87,03 & 11,60 & & \\
\hline \multicolumn{6}{|c|}{$\begin{array}{l}\text { Índice del Dominio de } \\
\text { Universalidad }\end{array}$} \\
\hline Masculino & 75,56 & 77,77 & 13,48 & 6462,0 &, 002 \\
\hline Femenino & 80,71 & 83,33 & 13,15 & & \\
\hline
\end{tabular}

$\overline{\text { Nota }}:=$ media, $M d n=$ mediana, $U=\mathrm{U}$ de Mann-Whitney, $p=$ significancia.

$=54,89, M d n=53,33)$ que en las mujeres $(=47,84, M d n$ $=46,66)$.

Prevalencias de consumo de alcohol y tabaco.

En relación al consumo de alcohol se mostró que un $89,9 \%$ (IC95\%; 86-94) de los participantes han consumido alcohol alguna vez en la vida, un 77,2\%(IC 95\%;72-82) ha consumido alcohol en el último año, 42,5\% (IC 95\%; 37 42) ha consumido alcohol en el último mes y un $28 \%$ (IC 95\%; 23-33) han consumido alcohol en los últimos 7 días. De acuerdo a las prevalencias del consumo de tabaco, se reporta que un 50,7\% (IC 95\%; 45-57) de los participantes han consumido tabaco alguna vez en la vida. $30,6 \%$ (IC $95 \% ; 25-36)$ ha consumido tabaco en el último año, el
23,1\% (IC 95\%; 18-28) ha consumido alcohol en el último mes y el 13,4 \% (IC 95\%; 09-18) han consumido alcohol en los últimos 7 días.

\section{Valores y consumo de alcohol.}

Para responder al objetivo que propone describir la relación del perfil de valores con el consumo de alcohol que presentan los jóvenes universitarios se utilizó el Coeficiente de Correlación de Spearman. Los resultados mostraron que solo existen correlaciones negativas y significativas, entre la sumatoria de AUDIT con el índice del dominio de conformidad $\left(\mathrm{r}_{\mathrm{s}}=-.203, \mathrm{p}<.003\right)$ es decir, que entre mayor sea el dominio de conformidad, menor es el consumo de alcohol. 
Tabla 4. Modelo de Regresión Lineal Backward los dominios de valores y el consumo de alcohol en el último año.

\begin{tabular}{lcrrrrr}
\hline $\begin{array}{l}\text { Modelo } \\
n=268\end{array}$ & \multicolumn{2}{l}{$\begin{array}{l}\mathrm{R}^{2}=16.5 \% \\
\text { Coeficiente de } \\
\text { variación }\end{array}$} & & \multicolumn{3}{c}{ Significancia } \\
\hline $\begin{array}{l}\text { Dominios } \\
\quad \text { Hedonismo } \\
\quad \text { Seguridad }\end{array}$ & B & EE & $\beta$ & $t$ & $p$ \\
&,- 053 &, 010 & 1,048 & 22,084 &, 001 \\
\hline
\end{tabular}

Tabla 5. Modelo de Regresión Lineal Backward los dominios de valores y el consumo de tabaco en el último año.

\begin{tabular}{lllll}
$\begin{array}{l}\text { Modelo } \\
n=268\end{array}$ & $\begin{array}{l}\mathrm{R}^{2}=11.4 \% \\
\text { Coeficiente de } \\
\text { variación }\end{array}$ & Significancia \\
\hline B E E & $\beta$ & $t$ & $p$
\end{tabular}

Dominios

\begin{tabular}{lrrrrr} 
Hedonismo &, 034 &, 009 & 1,035 & 13,437 &, 001 \\
Conformidad &,- 025 &, 011 &, 975 & 4,897 &, 027 \\
\hline
\end{tabular}

La tabla 4, se muestra que se aplicó un modelo de regresión logística con procedimiento Backward para identificar los dominios de valores que permanecían con efecto sobre el consumo de alcohol en el último año y se identificó que los valores de Hedonismo es el factor que presenta efecto en el consumo de alcohol en los jóvenes universitarios; sin embargo, el valor Seguridad es el factor que mantiene efecto en el no consumo de alcohol, con una varianza explicada de $\mathrm{R}^{2}=16,5 \%$.

La tabla 5, muestra que se aplicó un segundo modelo de regresión logística con procedimiento Backward para identificar los dominios de valores que permanecían con dicho efecto sobre el consumo de tabaco; y se identificó que el dominio Hedonismo es el factor que se mantiene presentando este efecto en el consumo de tabaco en el último año en los jóvenes universitarios; no obstante, el dominio Conformidad es el factor que se mantiene, presentando este efecto en el no consumo de tabaco en el último año en los jóvenes universitarios, con una varianza explicada de $11,4 \%$.

\section{DISCUSIÓN}

En relación con el perfil de valores de los jóvenes universitarios, los valores con mayor puntuación fueron el dominio de Conformidad, seguido de Benevolencia, logro, Universalismo e Individualidad. El orden de estos dominios son diferentes a los reportados en jóvenes Españoles de 12 a 18 años de edad, en donde se reportan las medias de mayor a menor importancia tales como; hedonismo seguido de la Estimulación, Individualidad, Benevolencia, Conformidad, Universalismo, Seguridad, Tradición, Logro y Poder (15).

Probablemente los resultados obtenidos en relación al orden de importancia que reportaron los participantes del presente estudio tenga que ver, con la edad y escolaridad del joven universitario, quien cursa una carrera de educación superior, con aspiraciones y retos de futuro quién busca en esta etapa, ser competente, tener éxito y piensa en un futuro próximo en la inserción activa en el mundo laboral y social como parte del desarrollo de sus valores (10). Así mismo se encuentra en plenitud de su desarrollo biológico y psíquico, donde se consolida el desarrollo de la personalidad y el carácter, son relativamente firmes y seguros, con todas las diferencias individuales que pueden darse en la realidad y está en capacidad de tomar sus propias decisiones de forma independiente.

Por su parte Díaz M, Castro D, y Cuevas B (16), refieren que la formación de valores profesionales, brinda una aproximación del cumplimiento del compromiso que tienen las Instituciones de Educación Superior con la sociedad, puesto que dentro de un mundo globalizado se tiene la obligación de tributar profesionales humanizados capaces de transformar la realidad, brindar soluciones adecuadas a cada situación, que consideren la realidad humana misma.

Se observó diferencia significativa de los valores por carrera, siendo mayor las medias de los valores en los universitarios de la carrera de enfermería, que en los universitarios de la carrera de ingeniería. Los dominios de valores que mostraron esta diferencia fueron: Individualismo, Conformidad, Tradición, Benevolencia y Universalidad. Estos resultados coincide con Montoya (17), quién en un estudio en universitarios reportó medias más altas en universitarios de Psicología (área de salud) que en los de administración, en los dominios de Benevolencia, Conformidad y Universalismo.

Probablemente las diferencias encontradas sea resultado de la formación específica que recibe el estudiante de la carrera de enfermería y otras del área de la salud. Los valores son fortalecidos y aunados a sus valores personales durante su formación en las instituciones formadoras de este profesional de nivel superior. Estos valores le dan la pauta para que el profesional de enfermería no pierda 
de vista que el ser humano con problemas de salud, tiene dignidad humana y que es, precisamente en esa circunstancia, cuando necesita de un profesional con valores bien cimentados, que conserve y fomente la dignidad de la persona ante la salud y la enfermedad (18).

Referente al perfil de valores en general por sexo se encontró diferencia significativa con media más alta para mujeres que para hombres. Así mismo se encontró diferencia significativa en cinco dominios de valor mostrando medias más altas en las mujeres a excepción del dominio poder que fue mayor en los hombres.

Esta diferencia encontrada probablemente se deba al conocimiento evolutivo que se tiene de la mujer como parte de la cultura, quien ha tenido un rol más social y menos competitivo que el hombre, además de su rol tradicional como conciliadora en las familias, lo que explica que las mujeres tienden a mostrar valores más sólidos que los hombres, menos dispuestas a traicionar, más honestas, francas y directas que el hombre (19). Respecto con la descripción de la relación del perfil de valores con el consumo de alcohol que presentan los jóvenes universitarios se encontró que existe correlación negativa y significativa en el dominio de conformidad con la puntuación del AUDIT es decir que a mayor valor de conformidad, menor es el consumo de alcohol. En este dominio incluye valores según Schwartz (9); buenos modales, autodisciplina, honrar a los padres y responsabilidad, que de alguna forma están influyendo para evitar el consumo de alcohol, este resultado coincide con resultados realizados en jóvenes españoles (20).

Referente al objetivo sobre determinar el efecto de los valores con las prevalencias del consumo de alcohol y tabaco en jóvenes universitarios, se encontró efecto significativo de los dominios de Hedonismo y de Seguridad con la prevalencia del consumo de alcohol en el último año. En relación con el consumo de tabaco se encontró efecto significativo en los dominios de Hedonismo y Conformidad con la prevalencia en el último año.

Estos resultados de alguna forma permiten documentar de manera empírica el perfil de valores a través de los dominios (9), que tienen los jóvenes universitarios, pudiendo determinar cuáles de ellos, influyen a través del efecto que presentan con el consumo de alcohol y tabaco a través de la medición de prevalencias de consumo. Permiten además identificar el efecto para el consumo y no consumo de estas sustancias, lo que se puede fundamentar según Schwartz y Bilsky (8) que los valores son metas deseables y trasituacionales incluidos en los dominios motivacionales que se valoran por el rango de importancia y que actúan como principios guía en la vida de cada persona. Estos valores van más allá de las acciones y situaciones específicas, sirven como estándares, guían la selección o evaluación de las acciones hacia los demás, se ordenan por la importancia relativa de unos sobre otros, forman un sistema relativamente estable y ordenado de las prioridades que lo caracterizan y son fundamentales para el autoconcepto.

Estos resultados se pueden analizar fundamentando que los valores están supeditadas a las percepciones, los intereses y los deseos de las personas en un contexto heterogéneo, lo que es valioso para unos no siempre lo es para otros (21). Se afirma que el valor es una cualidad estructural que surge de la reacción y relación de una persona frente a propiedades que se haya en un objeto. La relación física y humana determinada es posible por el conjunto de factores y eventos del contexto físico, social, cultural e histórico dentro de los cuales existe y tienen sentido los valores. Estos resultados permiten continuar estudiando los valores con mayor énfasis en los dominios de acuerdo a Schwartz en otras poblaciones de jóvenes universitarios de otras carreras y el consumo de alcohol y tabaco para profundizar el conocimiento en esta temática. Desarrollar en un futuro intervenciones de Enfermería para la prevención del consumo de alcohol y tabaco en jóvenes universitarios.

\section{REFERENCIAS BIBLIOGRAFICAS}

1. Moïsi D. L'Occident et l'Asie: innovation technologique et message universel. Paris: Le Monde; 2010. (Citado el 10 de octubre del 2014). Disponible en: http://lesclesdedemain. lemonde.fr/organisations/1-occident-et-1-asie-innovationtechnologique-et-message-universel_a-12-266.html

2. Instituto Nacional de Psiquiatría Ramón de la Fuente Muñiz, Instituto Nacional de Salud Pública, Secretaría de Salud. Encuesta Nacional de Adicciones 2011 Alcohol. México DF: INPRFM, INSP SS; 2012 (Citado el 10 de octubre del 2014). Disponible en: http://www.conadic.salud. gob.mx/pdfs/ENA_2011_ALCOHOL.pdf

3. Instituto Nacional de Psiquiatría Ramón de la Fuente Muñiz, Instituto Nacional de Salud Pública, Secretaría de Salud. Encuesta Nacional de Adicciones 2011 Tabaco. México DF: INPRFM, INSP, SS; 2012 (Citado el 10 de octubre del 2014). Disponible en: http://www.conadic.salud. gob.mx/pdfs/ENA_2011_TABACO.pdf

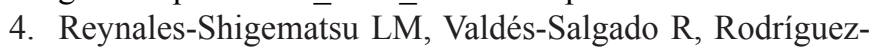
Bolaños R, Lazcano Ponce E, Hernández-Ávila M. Encuesta de tabaquismo en jóvenes en México:Análisis descriptivo 2003, 2005, 2006, 2008. Cuernavaca, México: InstitutoNacional de Salud Pública; 2009.

5. Organización Mundial de la Salud. Prevention is better than cure, say Romanian doctors. Bulletin of the World 
Health Organization. 2011; 89(4): 248-249.

6. Londoño C, García W, Valencia S, Vinaccia S. Expectativas frente al consumo de alcohol en jóvenes universitarios colombianos. Anales de Psicología. 2005; 21(2): 259-167.

7. Cano-Lozano M, Martín-Chaparro M, Vera-Martínez J. Preferencias de valores y consumo de tabaco y alcohol y otras drogas en jóvenes universitarios. Journal Health Psicology. 2003; 15 (1-2): 135-153.

8. Villatoro J, Medina-Mora M, Fleiz C, et al. El consumo de drogas en México: Resultados de la Encuesta Nacional de Adicciones. Salud Mental. 2011; 35(6): 287-297.

9. Schwartz S. Universals in the content and structure of values: Theoretical advances and empirical test in 20 countries. En: Zanna M (Ed.) Advances in experimental social psychology. New York: Academic Press; 1992.p. 1-65.

10. Schwartz S, Blinsky W. Toward a universal psychological structure of human values. Journal of Personality and Social Psychology. 1987; 53(3): 550-562.

11. Burns N, Grove SK. Investigación en Enfermería. 3a ed. Barcelona, España: Elsevier España S.L; 2004.

12. De la Fuente J, Kershenobich D. El alcoholismo como problema médico. Revista Facultad de Medicina UNAM. 1992; 35(2):47-51.

13. Babor TF, Higgins-Biddle JC, Saunders JB, Monteiro, MG. Cuestionario de Identificación de los Trastornos Debidos al Consumo de Alcohol. Ginebra, Suiza: Organización Mundial de la Salud; 2001.

14. Secretaria de Salud. Reglamento de la ley general de salud en materia de investigación para la salud. México DF:SSA; 1987.

15. Lezcano F, Abella V, Casado R. Implicaciones de la teoría de valores humanos de Schwartz en la actividad educativa con adolescentes. Revista Iberoamericana de Educación. 2012; 60(1): 1-15.
16. Díaz M, Castro D, Cuevas B. Valores profesionales de enfermería: Una mirada hacia la formación en la Educación Superior. Humanidades Médicas. 2012; 12(2): 289-299.

17. Montoya R. Comparación de los valores de los estudiantes de primer semestre de las facultades de Psicología y Administración de la Universidad de La Sabana. Tesis de Licenciatura en Psicología. Bogotá, Colombia: Universidad de la Sabana; 2012.

18. Lara R. El valor de los valores en la profesión de Enfermería. Especialización en Sociología de la Educación Superior; 2007.

19. Acevedo R. Hombres, mujeres y moral. Ñuñoa, Chile: La Tercera, Grupo COPESA; 2012.

20. Ramírez L, Musitu G. Relaciones entre los recursos y el consumo de alcohol y tabaco en adolescentes. Revista Española de Drogodependencia. 2008; 33 (1):53-66.

21. Schwartz S, Boehnke K. Evaluatingthestructure of human valueswithconfirmatory factor analysis. Journal of Research in Personality. 2004; 38: 230-255.

\section{Correspondencia:}

Rodríguez Aguilar Lucio

Correo electrónico: lucio.rodrigueza@uanl.mx

Fecha de Recepción: 05 de Agosto del 2015.

Fecha de aceptación: 11 de Agosto del 2015. 